\title{
Costo-beneficio de un programa preventivo y terapéutico para reducir la deficiencia de hierro en Argentina
}

\author{
Ignacio Drake ${ }^{1}$ y Ricardo Bernztein ${ }^{2}$
}

Forma de citar

Drake I, Bernztein R. Costo-beneficio de un programa preventivo y terapéutico para reducir la deficiencia de hierro en Argentina. Rev Panam Salud Publica. 2009;25(1):39-46.

RESUMEN Objetivos. Estimar los costos directos e indirectos de la deficiencia de hierro (DH) y la anemia ferropénica $(A F)$ en Argentina y compararlos con los costos de un programa específico para su prevención y tratamiento.

Métodos. Análisis de escenario previo (ex ante) de la relación costo-beneficio de un programa de prevención y tratamiento de la AF dirigido a todos los niños y las embarazadas pobres y sin cobertura social de Argentina. Las consecuencias económicas de la DH y la AF se estimaron a partir de los costos directos - gastos vinculados a la atención de un parto prematuro-e indirectos — pérdidas en la productividad futura de los niños por su peor desarrollo cognitivo debido a la DH y la menor productividad de los adultos por la AF- mediante la metodología específica desarrollada por The Micronutrient Initiative. Las intervenciones se definieron según las Guías de Práctica Clínica vigentes en Argentina y los costos de los componentes se tomaron de los precios de las licitaciones del Ministerio de Salud de la Nación.

Resultados. Cada US\$1,00 invertido en un programa de prevención y tratamiento de la DH y la AF, con una cobertura de $90 \%$ de la población de lactantes y embarazadas pobres sin seguro explícito de salud, representaría un ahorro de US\$33,40 por la prevención de las pérdidas económicas debidas a estas enfermedades.

Conclusión. Las intervenciones para enfrentar la DH no solo mejoran significativamente el estado de salud de la población, sino que representan un ahorro considerable de recursos.

Palabras clave Anemia, anemia ferropénica, deficiencia de hierro, suplementos dietéticos, alimentos fortificados, Argentina.

La deficiencia de hierro (DH) y su etapa final, la anemia ferropénica (AF), son las deficiencias nutricionales de

\footnotetext{
Programa Remediar, Ministerio de Salud de la Nación, Buenos Aires, Argentina. La correspondencia se debe dirigir a Ignacio Drake, Programa Remediar, Ministerio de Salud de la Nación, Calle Tomás Liberti No. $475,9^{\circ} \mathrm{D}$ (C1156AGB), Buenos Aires, Argentina. Correo electrónico: cidrake@hotmail.com

2 Hospital de Pediatría J. P. Garrahan y Programa Remediar, Ministerio de Salud de la Nación, Buenos Aires, Argentina. Correo electrónico: rbernz@ intramed.net
}

mayor prevalencia en el mundo y constituyen un importante problema de salud pública, en especial en los países en desarrollo (1). En el mundo, más de 2000 millones de personas sufren de alguna de estas deficiencias nutricionales; los lactantes y las embarazadas constituyen los grupos más vulnerables debido a la alta demanda de hierro durante el crecimiento infantil y el embarazo y las graves consecuencias que acarrea su déficit (2).
La AF se convierte en un problema mayor de salud pública cuando su prevalencia es superior a 5\% (3). De acuerdo con los resultados de la Encuesta Nacional de Nutrición y Salud (ENNyS) de 2005 (4), la prevalencia de anemia en Argentina es muy alta y alcanza 34,3\% ( $18,5 \%$ con $\mathrm{DH}$ y $15,8 \%$ sin ella) en niños de 6 a 23 meses; 8,9\% en niños de 2 a 5 años; $30,5 \%$ en embarazadas durante el primer trimestre; y $18,1 \%$ en mujeres no embarazadas de 10 a 49 años. La preva- 
lencia de $\mathrm{AF}$ es aún mayor en los grupos con mayores carencias económicas: en hogares con necesidades básicas insatisfechas (NBI), ${ }^{3}$ la prevalencia de AF en 2005 en la población infantil de 6 a 23 meses fue de $41,1 \%$, mientras que en embarazadas fue de $38,1 \%$ y en mujeres de 10 a 49 años, de 22,0\%. Del total de casos de AF encontrados en niños de 6 a 23 meses, $63,6 \%$ se clasificó como de grado leve, $35 \%$ moderado y $1,3 \%$ grave. De las mujeres que presentaron AF durante el primer trimestre de su embarazo, 9,1\% fue leve y 90,2\% moderada. Aunque según datos de la Organización Mundial de la Salud (OMS), la prevalencia de DH es 2,5 veces mayor que la de AF (3), en Argentina esta relación es cercana a 1:1 (4).

Desde una perspectiva epidemiológica, la AF y la DH tienen consecuencias sanitarias adversas con implicaciones económicas que pueden llegar a ser considerables. Tomando en cuenta la alta prevalencia de AF, sus graves efectos sobre la salud y la existencia de medidas efectivas para controlarla, se necesitan estudios que evalúen los costos de las intervenciones en relación con los beneficios que pueden generar. En este sentido, The Micronutrient Initiative, una organización canadiense sin fines de lucro, desarrolló en 1998 una metodología (6) que permite estimar, entre otros indicadores económicos, los costos que generaría la menor productividad futura de los niños con DH y AF debido a su peor desarrollo cognitivo. En 2003, Horton y Ross revisaron y actualizaron los métodos de estimación originales (7). Esta metodología se basa en valores estimados per cápita, costos unitarios, indicadores del mercado de trabajo y la prevalencia de AF y utiliza coeficientes de rendimiento que permiten cuantificar en términos económicos las consecuencias de la AF y la DH. Así, se puede valorar si los gastos de los programas de prevención o tratamiento de la DH y la AF tienen una relación costo-beneficio positiva al compararlos con los costos generados por la enfermedad en ausencia de esos programas.

El objetivo del presente trabajo fue estimar los costos directos e indirectos de

\footnotetext{
Se consideran hogares con NBI los que presentan al menos una de las siguientes condiciones: hacinamiento, vivienda de tipo inconveniente, malas condiciones sanitarias, no asistencia escolar de al menos un niño de 6 a 12 años o capacidad de subsistencia irregular (5).
}

la DH y la AF en Argentina y compararlos con los costos de un programa específico para su prevención y tratamiento.

\section{MATERIALES Y MÉTODOS}

Se realizó un análisis de escenario previo (ex ante) de la relación costo-beneficio de un programa de prevención y tratamiento de la AF a nivel nacional dirigido a niños y embarazadas pobres y sin cobertura social. Este programa está compuesto por dos subprogramas: el de prevención (fortificación de la leche con hierro) y el de tratamiento (complementación medicamentosa con sulfato ferroso). Para este análisis se tomaron en cuenta los costos directos e indirectos del tratamiento de la DH y la AF (8).

Se consideró un escenario ideal de cobertura de $90 \%$ de la población objetivo, tomada a partir de las estadísticas vitales publicadas por la Dirección de Estadísticas e Información de Salud (2004) y el Instituto Nacional de Estadísticas y Censos (INDEC), ambos de Argentina. Si bien los programas para enfrentar la $\mathrm{AF}$ deben ser integrales (9) —es decir, abarcar la promoción de la lactancia materna, la modificación de la dieta para aumentar el consumo de hierro y los facilitadores de su absorción, la fortificación de los alimentos básicos de la dieta con hierro, el suplemento de hierro en medicamentos, la prevención del bajo peso al nacer, la ligadura tardía del cordón umbilical y el tratamiento de las parasitosis, entre otras medidas-, para este estudio se tomó en cuenta solamente la fortificación de la leche y la complementación medicamentosa con sulfato ferroso.

En cuanto a la tasa de utilización de la leche y el hierro, se siguieron las recomendaciones de las Guías de Práctica Clínica del Ministerio de Salud de la Nación (2), según las cuales la prevención de la AF se debe basar en la fortificación de la leche con hierro, ácido ascórbico y zinc y el tratamiento de los lactantes y las embarazadas anémicos con sulfato ferroso. ${ }^{4}$ Para la prevención se consideró como población objetivo sin AF a $67 \%$ de los lactantes de 6 a 24 meses de edad. Cada lac-

\footnotetext{
4 Aunque la OMS recomienda la suplementación universal cuando la prevalencia de AF llega a $40 \%$ y en la práctica fuera poco probable que no se proveyera leche a quien se le prescribiera sulfato ferroso, para los efectos de este trabajo no se consideraron la fortificación de la leche y la complementación medicamentosa concurrentes.
}

tante entre los 6 meses y 1 año (un tercio de esta población objetivo) recibiría $2 \mathrm{~kg}$ de leche fortificada por mes y cada lactante de 13 a 24 meses de edad (dos tercios de la misma población objetivo) recibiría $1 \mathrm{~kg}$ de leche fortificada mensual; por tanto, cada mes se proveerían en promedio $1,3 \mathrm{~kg}$ por lactante, lo que equivale aproximadamente a $16 \mathrm{~kg}$ anuales. Por su parte, cada embarazada recibiría $10 \mathrm{~kg}$ de leche entre el quinto mes de embarazo y el parto. Para el tratamiento se consideraron los lactantes anémicos, $34 \%$ de la población, y que cada uno recibiría dos frascos de sulfato ferroso por mes entre los 6 meses y los 2 años de edad ( $3 \mathrm{mg} / \mathrm{kg}$ ) y las embarazadas, 30 comprimidos de hierro con ácido fólico mensuales entre el momento de su captación y los 6 meses posteriores al parto. Para el cálculo se consideró un tiempo de tratamiento de 18 meses para los lactantes y de 6 meses para las embarazadas.

Los costos de la leche fortificada y el sulfato ferroso se tomaron de las licitaciones realizadas por las instituciones nacionales pertinentes:

- leche fortificada: US\$3,00 por kg, según una licitación del Departamento Nacional de Salud Materno Infantil de 2005

- sulfato ferroso (solución de $12,5 \mathrm{~g} / 100 \mathrm{~mL}$ ): US\$ 0,20 por frasco de $20 \mathrm{~mL}$, según una licitación del Programa Remediar de 2004

- comprimidos de hierro (60 mg) y ácido fólico $(400 \mu \mathrm{g})$ : US\$ 0,70 por frasco de 30 unidades, según una licitación del Programa Remediar de 2004.

Según el Manual Farmacéutico Argentino de 2006, los precios de los medicamentos en las farmacias minoristas eran aproximadamente 10 veces mayores a los de estas licitaciones: el frasco de suspensión de sulfato ferroso costaba US\$2,00 y cada frasco de 30 comprimidos de hierro y ácido fólico costaba US\$ 6,70 (10).

Se estimó que los costos de administración y distribución de la leche y los medicamentos ascendían a $10 \%$ del costo de los productos. No se tomaron en cuenta los costos de los recursos humanos participantes en el programa, ya que formaban parte de los programas de control de los lactantes sanos y del embarazo, establecidos en todos los centros de atención primaria de la salud (Programa Remediar).

Para estimar las pérdidas económicas secundarias a la DH se siguió la metodo- 
logía utilizada por The Micronutrient Initiative, basada en el cálculo de los costos directos e indirectos (6). Los primeros abarcan los costos asociados con la atención de los partos prematuros, mientras los segundos se componen de la pérdida cognitiva y la disminución de la productividad por fatiga en la adultez. Para los costos indirectos se estiman coeficientes que se incorporan en los distintos cálculos monetarios para reflejar el peso que tiene la DH sobre el desenvolvimiento económico individual, con especial referencia al mercado de trabajo y la contribución per cápita al producto interno bruto (PIB). Esto permitió cuantificar el efecto de la DH sobre la productividad en el trabajo y la capacidad de generar ingresos.

Se debe subrayar que la estimación realizada de los costos directos e indirectos fue conservadora, ya que no se tomaron en cuenta los costos derivados de la pérdida de vidas maternas o infantiles, infecciones, etc., por no disponer de una metodología validada para hacerlo.

Los resultados se presentan en dólares estadounidenses per cápita, en valores absolutos y como porcentaje del PIB. Se calculó también la razón entre ambos costos.

\section{Análisis de sensibilidad}

El análisis de la relación costo-beneficio trae aparejado cierto margen de incertidumbre debido a que en ocasiones no se cuenta con información metodológicamente comparable o existen fuertes diferencias entre los nomencladores para estimar los costos (por ejemplo, el costo de un parto). Para este trabajo se mantuvieron fijos los valores provenientes de fuentes confiables, como las estadísticas vitales (número de nacidos vivos y proporción de niños nacidos antes de la semana 37 de gestación), las encuestas permanentes de hogares (participación del salario en el PBI, participación de los asalariados manuales y de los trabajadores manuales dedicados a actividades pesadas) y las cuentas nacionales (PBI per cápita), por considerar que son fuentes con una metodología de medición validada y que no presentan sesgos importantes. No obstante, la adaptación de esta metodología a las condiciones argentinas puede provocar alteraciones en los coeficientes básicos utilizados. Para conocer el efecto de estas variaciones se realizó un análisis de sensibilidad para los valores que no provenían de fuentes sistemáticas o cuyos valores de

CUADRO 1. Población diana y costo anual de un programa de prevención y tratamiento de la anemia ferropénica en Argentina ${ }^{a}$

\begin{tabular}{lccc}
\hline \multicolumn{1}{c}{ Subprograma } & \multicolumn{3}{c}{ Población diana } \\
\cline { 2 - 3 } & Lactantes & Embarazadas & Total \\
\hline Prevención & & & \\
Población (miles) & 335,0 & 190,4 & 525,4 \\
Cobertura del programa de 90\% (miles) & 301,5 & 171,3 & 472,8 \\
Consumo de leche per cápita (kg/año) & 16,0 & 10,0 & 26,0 \\
Costo de la leche (US\$/kg) & 3,00 & 3,00 & 3,00 \\
Costo total (millones de US\$año) & 14,5 & 5,1 & 19,6 \\
Tratamiento & 165,0 & 89,6 & 254,6 \\
Población (miles) & 148,5 & 80,6 & 229,1 \\
Cobertura del programa de 90\% (miles) & 24 & 12 & $\mathrm{NA}$ \\
Consumo del hierro per cápita (tratamientos/año) & 0,20 & 0,70 & $\mathrm{NA}$ \\
Costo del suplemento ferroso (US\$/frasco) & 0,7 & 0,7 & 1,4 \\
Costo total (millones de US\$año) & & & 780,0 \\
Total del programa & 500,0 & 280,0 & 702,0 \\
$\quad$ Población (miles) & 450,0 & 252,0 & 21,0 \\
Cobertura del programa de 90\% (miles) & 15,2 & 5,8 & \\
Costo anual (millones de US\$) & & & \\
\hline
\end{tabular}

Fuente: Elaborado a partir de datos de la Encuesta Nacional de Nutrición y Salud (4) y del Instituto Nacional de Estadísticas y Censos, de Argentina, y los precios de licitación provistos por el Departamento Nacional de Salud Materno Infantil y el Programa Remediar, 2004 (11).

a Se consideró una cobertura de $90 \%$ de la población objetivo.

b NA: no aplica.

referencia presentaran una gran variabilidad (incremento proporcional del gasto de atención de un parto prematuro, riesgo de prematuridad debido a $\mathrm{DH}$ en la población, costo de la atención de un parto, pérdida cognitiva, prevalencia de anemia en niños, pérdida laboral en el sector de asalariados manuales, pérdida laboral en el sector de trabajos pesados y prevalencia de anemia en adultos).

\section{RESULTADOS}

\section{Costos del programa de prevención y tratamiento}

La posible población objetivo de un programa de prevención y tratamiento de la AF en todo el país en el año 2005 era de aproximadamente 500000 lactantes de 6 meses a 2 años de edad y 280000 embarazadas pobres y sin seguro explícito de salud. Para los cálculos de costos se consideró una cobertura ideal de $90 \%$, equivalente a 450000 lactantes entre 6 y 24 meses de edad y 252000 embarazadas.

El subprograma de prevención de la DH cubriría a 301500 lactantes de 6 a 24 meses de edad sin AF, de los cuales 100500 niños de 6 a 12 meses de edad (una tercera parte de la población objetivo) recibirían $2 \mathrm{~kg}$ mensuales de leche fortificada y 200100 lactantes de 13 a 24 meses de edad (dos tercios de la población objetivo) recibirían $1 \mathrm{~kg}$ mensual,
Esto equivale a $4824000 \mathrm{~kg}$ de leche fortificada anuales, es decir, un promedio total de $16 \mathrm{~kg}$ anuales por lactante cubierto. Mientras, cada embarazada $\mathrm{cu}-$ bierta recibiría en promedio $10 \mathrm{~kg}$ de leche fortificada, para un costo total de $1713600 \mathrm{~kg}$ anuales. El costo total de este subprograma sería de US\$ $14,5 \mathrm{mi}-$ llones al año para los lactantes y US\$ 5,1 millones al año para las embarazadas (cuadro 1).

En cuanto al subprograma de tratamiento de la $\mathrm{AF}$, cada lactante anémico recibiría en promedio 24 frascos anuales de suspensión de sulfato ferroso, equivalente a un total de US\$ 0,7 millones al año. Cada embarazada o puérpera recibiría anualmente hasta 12 envases con 30 comprimidos, ello equivale también a un total de US\$ 0,7 millones al año.

El costo anual del Programa - tanto del subprograma preventivo como del terapéutico - para lactantes y embarazadas fue de US\$21,0 millones. Cabe señalar que la división de los subprogramas preventivo y terapéutico es una simulación, ya que si todos los niños de 6 a 24 meses recibieran las dotaciones de leche fortificada durante los primeros 18 meses de vida, una parte de los lactantes anémicos recibiría también la intervención preventiva y, por otra parte, otros lactantes que nacen y desarrollan AF en un mismo año recibirían únicamente 12 frascos de hierro durante ese período. 


\section{Costos directos e indirectos de la $\mathrm{DH}$ y la AF}

Los costos directos derivados de la atención de los partos prematuros vinculados a la DH y la AF se cuantificaron monetariamente según la siguiente fórmula (6):

Costo por prematuridad $=\mathrm{PrPpr} \times \mathrm{PrDH}$ $\times$ Nnacim $\times$ PrPrem $\times$ CParto

donde,

PrPpr = incremento proporcional del gasto de atención de un parto prematuro $\operatorname{PrDH}=$ riesgo de prematuridad debido a la $\mathrm{DH}$ en la población ${ }^{5}$

Nnacim $=$ número de niños nacidos vivos PrPrem $=$ proporción de niños nacidos antes de la semana 37 de gestación ${ }^{6}$ CParto $=$ costo de la atención de un parto. ${ }^{7}$

Al aplicar los valores estimados de cada factor, se obtuvo:

Costo por prematuridad $=100 \times 0,3594 \times$ $700000 \times 0,0756 \times$ US\$ $170=$ US\$323,3 $\times 10^{6}$

El ahorro de US\$ 323,3 millones que se obtendría por la prevención de los partos prematuros debido a AF y DH equivale a 0,35\% del PIB del año 2005 de Argentina.

En cuanto a los costos indirectos, la DH infantil tiene consecuencias irreversibles sobre la productividad en el ámbito laboral durante la vida adulta de los niños afectados. Según algunos estudios, se estima que la reducción en la capacidad de generar ingresos sería de alrededor de $4 \%$ (6). La fórmula empleada para conocer los costos indirectos vinculados con la pérdida cognitiva provocada por la AF y la DH fue:

Costos por pérdida cognitiva $=4 \% \times$ PaSal $\times$ PIB per cápita $\times$ PrAI

donde,

\footnotetext{
5 Este coeficiente se calculó en base a la relación entre la prevalencia de anemia en embarazadas (Pr) y el riesgo relativo (RR) de prematuridad por causa de la AF (estimado en 2,7). El valor se obtiene mediante la siguiente fórmula: $\operatorname{PrDH}=[\operatorname{Pr} \times$ $(\mathrm{RR}-1) / 1]-[\operatorname{Pr} \times(\mathrm{RR}-1)]$.

6 Se tomó como el porcentaje de recién nacidos con peso $<2500 \mathrm{~g}$. Se asumió que la mayor parte de los neonatos con bajo peso al nacer son prematuros.

7 Existen diversos criterios para calcular el costo de la atención de un parto. Este valor se estimó como US\$170,00, a partir de la información provista por informantes clave.
}

PaSal $=$ participación del salario en el PIB, a precios básicos (11)

PIB per cápita $=$ monto del PIB per cápita $(\text { en US\$) })^{8}$

PrAI = Prevalencia de anemia infantil.

Al aplicar los valores estimados de cada factor, se obtuvo:

Costos por pérdida cognitiva $=4 \% \times$ $28,5 \% \times$ US\$ $2363,6 \times 33 \%=$ US\$ 8,9

De esta manera, los costos indirectos asociados con la pérdida cognitiva serían de US\$ 8,9 per cápita, lo que ascendería a US\$ 343,2 millones de dólares en todo el país, es decir 0,38\% del PIB de Argentina en el año 2005. Este valor corresponde a las pérdidas cognitivas permanentes provocadas por haber padecido de AF durante la niñez, asumiendo que la prevalencia de AF en este grupo de edad ha permanecido estable en las últimas décadas.

Los costos indirectos debidos a la pérdida de productividad en los trabajadores adultos por fatiga física o muscular por la DH que se sufre son diferentes en los trabajadores asalariados manuales (5\% de pérdida) y en los que realizan trabajos manuales considerados pesados (12\% de pérdida). La fórmula empleada (6) fue:

Costos por pérdida laboral por fatiga física o muscular $=[5 \%($ PaSal $\times$ PaTAM $\times$ PIB per cápita $\times$ Pra $)]+[12 \%($ PaSal $\times$ PaTAMP $\times$ PIB per cápita $\times$ Pra) $]$

donde,

PaSal = participación del salario en el PIB, a precios básicos (11)

PaTAM = participación de los asalariados manuales 9

PaTAMP = participación de los trabajadores manuales en actividades pesadas ${ }^{10}$ PIB per cápita $=$ monto del PIB per cápita (en US\$)

Pra $=$ Prevalencia de anemia en adultos ${ }^{11}$

\footnotetext{
8 Estimación a partir del producto interno bruto de 2005, a precios del productor de 1993, en millones de dólares. INDEC. http://www.indec.mecon.ar/ nuevaweb/cuadros/17/cuadro15.xls

9 Se consideró la participación de los obreros o empleados sobre el total de trabajadores, según la Encuesta Permanente de Hogares del INDEC en el segundo semestre 2005. Elaboración propia.

10 Se consideró esta participación como 50\% del PIB generado por los sectores agricultura y construcción.

11 Se consideró como la mitad de la prevalencia de anemia en mujeres en edad fértil.
}

Al aplicar los valores estimados de cada factor, se obtuvo:

Costos por pérdida laboral por fatiga física o muscular $=[5 \% \times 28,5 \% \times 16,4 \% \times$ US\$ $2363,6 \times 8,5 \%]+[12 \% \times 28,5 \% \times$ $6,1 \% \times$ US\$ $2363,6 \times 8,5 \%$ ] = US\$ $0,47+$ US\$ $0,42=$ US $\$ 0,89$

Así, los costos indirectos vinculados con la fatiga física o muscular serían de US\$ 0,89 per cápita, que al llevar al total de la población del país asciende a US\$ 34,3 millones, equivalente a 0,03\% del PIB de Argentina en 2005.

En conjunto, ambos factores - las pérdidas cognitivas irreversibles y la fatiga física o muscular - generaron costos indirectos por un total de US\$ 9,8 per cápita, equivalente a US $\$ 377,5$ millones si se extrapola a la población del país, lo que representaría 0,41\% del PIB de Argentina en 2005.

En el cuadro 2 se presentan los costos totales (ingresos perdidos) vinculados a la $\mathrm{DH}$ y la AF. La relación entre el costo de las intervenciones para enfrentar la $\mathrm{DH}$ y la AF (US\$21,0 millones) respecto a los costos asociados con la enfermedad (prematuridad y bajo peso al nacer) y los ingresos dejados de percibir en términos de productividad por trastornos cognitivos y físico-musculares asociados con la DH y la AF (US\$ 700,8 millones) fue de $1 / 33,4$.

\section{Análisis de sensibilidad}

Según el estudio de sensibilidad basado en los costos directos (a partir de los costos de la atención de un parto y el incremento proporcional del gasto para la atención de un parto prematuro), los costos indirectos (a partir de la prevalencia de AF en niños y adultos y los coeficientes de rendimiento, tanto por la pérdida cognitiva como por la fatiga física o muscular) y los costos del programa de prevención y tratamiento, los costos y los beneficios solo se igualarían (se anularían) si los componentes utilizados para el cálculo se redujeran en $78 \%$ de manera simultánea. Con una reducción de $25 \%$, la relación sería de $1 / 16,6$ y con una reducción de $50 \%$ la relación sería de $1 / 6,4$, en ambos casos a favor de la intervención. Este análisis confirma la pertinencia del cálculo realizado y el fuerte impacto económico que tendría un programa de prevención y tratamiento de la DH y la AF (cuadro 2). 
CUADRO 2. Costos calculados y resultados del análisis de sensibilidad según dos alternativas de disminución de la relación costo-beneficio, ${ }^{a}$ Argentina, 2005

\begin{tabular}{|c|c|c|c|c|c|c|}
\hline \multirow[b]{2}{*}{ Tipo de costos } & \multicolumn{2}{|c|}{ Costos calculados } & \multicolumn{2}{|c|}{$\begin{array}{c}\text { Costos si los } \\
\text { components } \\
\text { disminuyen } 25 \%\end{array}$} & \multicolumn{2}{|c|}{$\begin{array}{c}\text { Costos si los } \\
\text { componentes } \\
\text { disminuyen } 50 \%\end{array}$} \\
\hline & Valorb & $\begin{array}{c}\% \text { del } \\
\text { producto } \\
\text { interno bruto }\end{array}$ & Valor ${ }^{b}$ & $\begin{array}{c}\% \text { del } \\
\text { producto } \\
\text { interno bruto }\end{array}$ & Valorb & $\begin{array}{c}\% \text { del } \\
\text { producto } \\
\text { interno bruto }\end{array}$ \\
\hline Directos & 323,3 & 0,35 & 136,4 & 0,15 & 40,4 & 0,04 \\
\hline Indirectos & 377,5 & 0,41 & 212,4 & 0,23 & 94,4 & 0,10 \\
\hline Totales & 700,8 & 0,77 & 348,8 & 0,38 & 134,8 & 0,15 \\
\hline Relación costo-beneficio & \multicolumn{2}{|c|}{$1: 33,4$} & \multicolumn{2}{|c|}{$1: 16,6$} & \multicolumn{2}{|c|}{$1: 6,4$} \\
\hline
\end{tabular}

a El costo total calculado de un programa de prevención y tratamiento de la anemia ferropénica en Argentina fue de US\$21,0 millones.

${ }^{b}$ En millones de dólares estadounidenses.

\section{DISCUSIÓN}

La prevalencia de AF en Argentina es elevada y no guarda relación con sus indicadores de desarrollo social. La DH y la AF - y sus consecuencias- constituyen un problema de salud pública que afecta a un gran número de personas a pesar de ser prevenibles, por lo que debe corregirse. El presente análisis parte del supuesto de que las intervenciones dirigidas a enfrentar la $\mathrm{DH}$ y la AF podrían reducir sus prevalencias a niveles muy bajos, similares a los observados en los países desarrollados.

Las consecuencias sanitarias de la $\mathrm{DH}$ y la AF son devastadoras y por sí mismas justificarían la implementación de programas específicos para enfrentarlas. El impacto económico de tales programas, a partir de análisis de la relación costo-beneficio como el presentado aquí, demuestra que los recursos utilizados en la implementación de estos programas no solo aportan beneficios a la salud, sino que redundan en un ahorro considerable en términos económicos para el sistema sanitario. Este análisis costobeneficio demostró que por cada dólar destinado a estos programas se generaría un ahorro de US\$33,40 por concepto de ahorro o aumento de la productividad (figura 1). Estos recursos pueden recuperarse a corto (disminución de la prematuridad), mediano (efectos de la fatiga) y largo (mejoría en el desarrollo cognitivo y la productividad) plazos.

Más allá de medir el impacto sanitario de las intervenciones para enfrentar la $\mathrm{DH}$, este trabajo documenta su eficiencia económica a partir de la relación entre los costos de las intervenciones preventi-
FIGURA 1. Relación entre los costos de un programa nacional de prevención y tratamiento de la anemia ferropénica y los costos directos e indirectos generados por la deficiencia de hierro por persona, Argentina

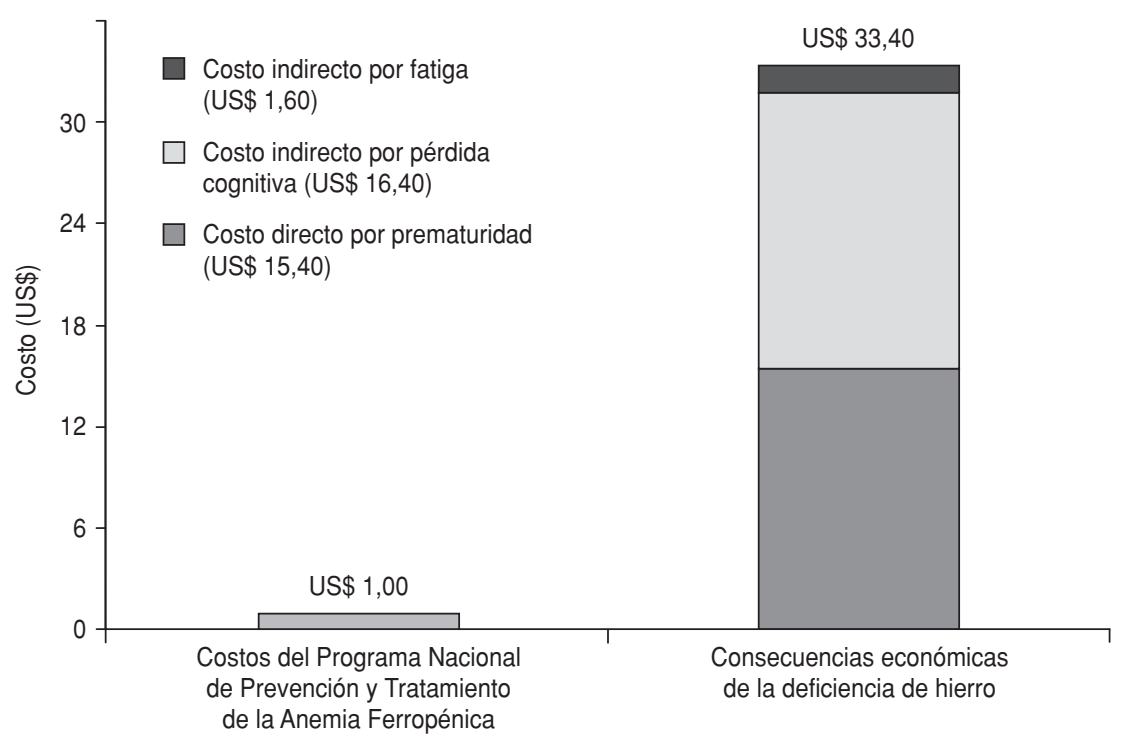

vas y terapéuticas y los beneficios para la sociedad.

Si bien la hipótesis inicial del trabajo era que el gasto en salud generaría ahorros económicos, sobre todo por tratarse de un trastorno de alta prevalencia con consecuencias muy negativas, resulta sorprendente la magnitud de la relación encontrada, especialmente tomando en cuenta que se basaron en estimados conservadores.

Los costos de los medicamentos comprados de modo centralizado (como en el programa Remediar) en comparación con los precios minoristas constituye un ejemplo de eficiencia de los programas nacionales debido a la economía de escala (12). Según las estimaciones realizadas para el año 2005, el costo de la DH en Argentina equivalía a 0,77\% del PIB, menor que el valor de 4,5\% encontrado por Horton y Ross en 10 países en desarrollo para el año 2003 (6) y del costo estimado de la deficiencia de vitaminas y minerales de 2,4-5,0\% del PIB en los países en desarrollo a mediados de la década de $1990(13,14)$.

Aunque se ha estudiado a profundidad el metabolismo del hierro, aún no se conocen totalmente algunas consecuen- 
cias de su deficiencia sobre la salud y no se debate suficientemente sobre el impacto negativo de la $\mathrm{DH}$ y la $\mathrm{AF}$ sobre la productividad del trabajo físico debido a la disminución de la resistencia al ejercicio muscular (15). También hay consenso acerca de los efectos nocivos de la $\mathrm{DH}$ y la AF sobre el desarrollo cognitivo y motriz mediados por alteraciones durante la neurogénesis y la maduración del sistema nervioso en el feto y los primeros 3 años de vida $(16,17)$. Sin embargo, la mayoría de los trabajos de investigación sobre el empleo de suplementos de hierro se limitan a medir el aumento en la concentración de hemoglobina como resultado primario y faltan estudios de mayor calidad (ensayos clínicos aleatorizados y controlados o metaanálisis) que analicen a partir de datos experimentales la relación entre los tratamientos con hierro, por una parte, y las consecuencias del parto, el desarrollo mental del lactante y la mortalidad materna y perinatal, por la otra.

En una revisión sistemática publicada recientemente por la Colaboración Cochrane que abarcó a 12706 mujeres, se concluyó que la complementación antenatal sistemática con hierro - diaria o semanal- podría ser benéfica en países con prevalencias elevadas de $\mathrm{DH}$ pregestacional, pero que se necesitaban más investigaciones, fundamentalmente en países en desarrollo, para llegar a una conclusión válida acerca de su efectividad y seguridad (18). Revisiones sistemáticas realizadas para evaluar el efecto de la complementación con hierro en el desarrollo mental o motor en niños encontraron que esa complementación era eficaz para reducir la prevalencia de AF como resultado primario, pero que faltan evidencias convincentes para evaluar su efecto en lactantes $(16,19)$.

Debido a la escasa evidencia científica para sostener o refutar la relación entre la DH y algunos trastornos de salud, la OMS realizó una evaluación comparativa de riesgos sanitarios con el propósito de superar la debilidad de esas evidencias (20). Estudios observacionales prospectivos y de evaluación comparativa de riesgos sanitarios encontraron una relación causal entre la DH - y la AF- y el aumento de la mortalidad materna, perinatal e infantil; el aumento en la frecuencia de la prematuridad y el bajo peso al nacer; el retardo en el desarrollo cognitivo e intelectual de los lactantes, que persiste por más de 10 años después del trata- miento; la disminución de la capacidad de trabajo; y el incremento del riesgo de padecer infecciones por déficit inmunitario $(21,22)$. La OMS ha informado que la AF está entre los 10 primeros factores de riesgo de años perdidos de vida saludable y que contribuye sustancialmente a la mortalidad y la discapacidad (23).

La mayor parte de la carga de enfermedad por DH se debe a su alta prevalencia en embarazadas y madres lactantes en países en vías de desarrollo. La complementación con hierro y ácido fólico aparece como una de las estrategias prioritarias en países con alta prevalencia de $\mathrm{AF}$, a pesar de que es difícil que una sola intervención logre reducir la tasa de mortalidad materna (24). Por ello, es legítimo realizar la siguiente pregunta: ¿cuánta evidencia se necesita para diseñar políticas y equipar programas cuando las investigaciones no son definitorias? A pesar de las limitaciones en que se basan las estimaciones del impacto sanitario asociado con la AF y la DH, hay consenso científico internacional, avalado por la OMS, de que es válido proyectar efectos sanitarios hacia el ámbito económico $(3,6)$.

Una limitación de este tipo de trabajo es la falta de "evidencia irrefutable" que respalde la eficacia, efectividad y costos de las intervenciones nutricionales para prevenir y controlar algunos efectos funcionales de la AF. Otra limitación es que, como todos los demás modelos empleados para estimar las consecuencias económicas de la AF, el propuesto por Horton y Ross para The Micronutrient Initiative se encuentra aún en un proceso de validación externa que permitirá avalar su aplicación en diferentes escenarios. Hay dificultades para capturar objetivamente las interacciones entre la nutrición, la salud y el desarrollo económico mediante la ponderación del peso de cada variable, por lo que han surgido múltiples modelos para estimar el costo económico de las deficiencias de vitaminas o minerales (hambre oculta) (25).

Se debe tener en cuenta que, según un análisis de las prescripciones del Programa Remediar, hay una baja frecuencia de atención de niños sanos, mujeres embarazadas o actividades preventivas (26), se subutilizan los suplementos de hierro (27) y existe una gran variabilidad en la práctica clínica entre las diferentes provincias del país y aun dentro de una misma provincia. No obstante, en esta investigación se planteó un escenario de cobertura ideal de $90 \%$ de la población obje- tivo y costos mínimos de los insumos. El bajo nivel de cobertura y la mala calidad de la atención que se presta en instalaciones del primer nivel de salud constituyen otra limitación de esta investigación.

Es importante también señalar las grandes dificultades encontradas para obtener la información confiable necesaria para calcular algunos costos, en especial los referidos a los gastos de la atención de los partos - existen numerosos y muy diversos nomencladores de programas nacionales y obras sociales, que no están centralizados y al alcance del público en general- lo que dificultó estimar con mayor precisión, entre otros, el incremento de los costos asociados con la atención de los partos prematuros. Esta falta de información se resolvió mediante entrevistas con informantes clave.

En esta investigación no se estimó el efecto de no haber contado dos veces a un mismo lactante - presentara AF o no- que estuviera recibiendo suplemento medicamentoso y leche fortificada. Para mantener una relación adecuada costo-beneficio en el sector social se necesitaría algún modo simple de diferenciar los grupos de la población en los que se deben implementar medidas preventivas o terapéuticas para la $\mathrm{DH}$. Eso requeriría un método de tamizaje sistemático, especialmente cuando la prevalencia de $\mathrm{AF}$ se encuentre entre 5\% y $20 \%$. Cuando la prevalencia de AF es de $40 \%$ en algún grupo de edad - como el segmento de lactantes pobres o indigentes que asisten a centros de atención primaria de salud- el enfoque más efectivo, y posiblemente más eficiente, sería la complementación universal del grupo en cuestión (3). La OMS no emite recomendaciones específicas para los casos en que la prevalencia de AF se encuentre entre $20 \%$ y $40 \%$.

Desde 2002, el Programa Remediar ha provisto la complementación o fortificación con hierro para los niños y las embarazadas en Argentina y desde 2003 la Dirección Nacional de Maternidad e Infancia provee leche fortificada con hierro, ácido ascórbico y zinc. Ya a partir de 2004 se implementó por ley la fortificación de la harina de trigo con hierro y ácido fólico. Esto hizo que en lugar de plantear la alternativa de prevención o tratamiento, se planteara implementar ambas medidas simultáneamente. Estas acciones son necesariamente complementarias y tienen diferentes tiempos, tanto para su implementación como para 
la observación de su impacto en la población. Mientras la modificación de la dieta opera a largo plazo, la fortificación de alimentos es eficaz a mediano plazo y la complementación con medicamentos da resultados a corto plazo en las personas con $\mathrm{AF}$ en el grupo poblacional en riesgo. La implementación coordinada de todas las estrategias pertinentes debe llevar a la disminución de la prevalencia de la AF y de la proporción de la población que requeriría tratamiento. También es necesario destacar que la fortificación de la leche, de mayor costo que la complementación con hierro, no solo previene la $\mathrm{DH}$, sino que también puede emplearse para proveer otros nutrientes.

Estos resultados demuestran que cada US\$ 1,00 invertido en un programa de prevención y tratamiento de la $\mathrm{DH}$ y la $\mathrm{AF}$, con una cobertura de $90 \%$ de la población de lactantes y embarazadas pobres sin seguro explícito de salud, representaría un ahorro de US $\$ 33,40$ por la prevención de las pérdidas económicas debidas a estas enfermedades. Esta información debe contribuir a adoptar decisiones políticas informadas sobre la más efectiva asignación de los recursos del presupuesto de salud pública en este campo. Se recomienda a los decisores políticos realizar las intervenciones necesarias para enfrentar la $\mathrm{DH}$ y la $\mathrm{AF}$, ya que además de mejorar significativamente el estado de salud de la población, representa un ahorro sustancial de recursos.

Agradecimientos. Se agradece a Erick Boy, de The Micronutrient Initiative, por sus aportes científicos y sus opiniones constructivas. Este trabajo se presentó en el II Congreso de Economía de la Salud de América Latina y el Caribe el 31 de octubre de 2006, en Buenos Aires, Argentina.

\section{REFERENCIAS}

1. International Nutritional Anemia Consultive Group. Anemia prevention and control: what works. Pt. 1-2. Washington, D.C.: USAID; 2003. Hallado en http://www.phnip.com/ portfolio/pubs.html. Acceso el 17 de octubre de 2008.

2. Calvo E, Longo E, Aguirre P, Britos S. Prevención de anemia en niños y embarazadas en la Argentina. Actualización para equipos de salud. Buenos Aires: Ministerio de Salud; 2001.

3. UNICEF, UNU, WHO. Iron deficiency anaemia. Assessment, prevention, and control. A guide for programme managers. Geneva: WHO; 2001. Hallado en http://www. who.int/nutrition/publications/en/ida assessment_prevention_control.pdf. Acceso el 4 de noviembre de 2008.

4. Ministerio de Salud de la Nación. Encuesta Nacional de Nutrición y Salud. Buenos Aires: MSAL; 2007. Hallado en http://www.msal. gov.ar/htm/Site/ennys/site/default.asp. Acceso el 17 de octubre de 2008.

5. Instituto Nacional de Estadísticas y Censos. Mapa de necesidades básicas insatisfechas 2001. Aquí se Cuenta (Buenos Aires). 2003; (7):1-2. Hallado en http://www.indec.gov. ar/webcenso/aquisecuenta/Aqui7.pdf. Acceso el 11 de noviembre de 2008.

6. Ross J, Horton S. Economic consequences of iron deficiency. Ottawa: The Micronutrient Initiative; 1998

7. Horton S, Ross J. The economics of iron deficiency. Food Policy. 2003;28:51-75.

8. Velazco M, Rodríguez-Monguió R. Análisis coste-beneficio: metodología y utilidad en la toma de decisiones sanitarias. En: DomínguezGil Hurlé A, Soto Álvarez J. Farmacoeconomía e investigación en salud: principios y práctica. Situación actual y perspectivas futuras en España. Madrid: Real Academia Nacional de Farmacia; 2002.

9. Freire WB, Kahn SG, McGuire J, Post GL, Interagency Anemia Steering Group. Anemia prevention and control: what works. Pt 1. Program guidance. Washington, D.C.: USAID; 2003. Hallado en http://www.phnip.com/ portfolio/pubs.html. Acceso el 17 de octubre de 2008.

10. Programa Remediar. Estimación de la eficiencia en la compra de medicamentos del Pro- grama Remediar. Atención primaria de la salud. Boletín PROAPS-REMEDIAR. 2004; 2(15):16. Hallado en http://www.remediar. gov.ar / ACM-Remediar-Publicaciones1. nsf/PubBoleWeb/1D38A9C84570D54303257 26E0069CC25/\$File/Boletin 15.pdf. Acceso el 17 de octubre de 2008.

11. Lindenboim J, Graña JM, Kennedy D. Distribución funcional del ingreso en Argentina. Ayer y hoy. Buenos Aires: Universidad de Buenos Aires; 2005. (Documento de Trabajo No. 4).

12. Programa Remediar. Estimación de la eficiencia en la compra de medicamentos del Programa Remediar. Atención primaria de la salud. Bol PROAPS-REMEDIAR. 2004;2(15): 16. Hallado en http://www.remediar.gov. ar/ACM-Remediar-Publicaciones1.nsf/Pub BoleWeb/1D38A9C84570D5430325726E0069 CC25/\$File/Boletin 15.pdf. Acceso el 17 de octubre de 2008.

13. Banco Mundial. Enriching lives: overcoming vitamin and mineral malnutrition in developing countries. Washington, D.C.: World Bank; 1994.

14. Murray CJL, López AD, eds. The global burden of disease: a comprehensive assessment of mortality and disability from diseases, injuries and risk factors in 1990 and projected to 2020. Cambridge, MA: Harvard University School of Public Health; 1996. (Global Burden of Disease and Injury Series Vol. 1.)

15. Haas JD, Brownlie T. Iron deficiency and reduced work capacity: a critical review of the research to determine a causal relationship. J Nutr. 2001;131:S676-90.

16. Beard JL. Iron biology in immune function, muscle metabolism and neuronal functioning. J Nutr. 2001;131:S 568-80.

17. Grantham-McGregor S, Ani C. A review of studies on the effect of iron deficiency on cognitive development in children. J Nutr. 2001; 131:S649-66.

18. Pena-Rosas JP, Viteri FE. Effects of routine oral iron supplementation with or without folic acid for women during pregnancy. Cochrane Database Syst Rev. 2006(3).

19. Sachdev H, Gera T, Nestel P. Effect of iron supplementation on mental and motor development in children: systematic review of ran- domised controlled trials. Public Health Nutr. 2005;8(2):117-32.

20. Ezzati M, Lopez AD, Rodgers A, Murray C, eds. Comparative quantification of health risks global and regional burden of disease attributable to selected major risk factors. Geneva: World Health Organization; 2004.

21. Stoltzfus RJ, Mullany L, Black R. Iron deficiency anaemia. In: Ezzati M, Lopez AD, Rodgers A, Murray C, eds. Comparative quantification of health risks: global and regional burden of disease attributable to selected major risk factors. Geneva: World Health Organization; 2004.

22. Lozoff B. Behavioral and developmental effects of preventing iron-deficiency anemia in healthy full-term infants. Pediatrics. 2003;112: 1-9.

23. World Health Organization. World Health Report 2002. Geneva: WHO; 2002.

24. Campbell OMR, Graham WJ, The Lancet Maternal Survival Series Steering Group. Strategies for reducing maternal mortality: getting on with what works. Maternal Survival 2. Lancet. 2006;368:1284-99.

25. Stein AJ, Qaim M. The human and economic cost of hidden hunger. Food Nutr Bull. 2007; 28(2):125-34

26. Bernztein R, Monsalvo M. Análisis de los diagnósticos o problemas de salud. Atención primaria de salud. Bol PROAPS-REMEDIAR. 2004;2(15):11-4. Hallado en http://www. remediar.gov.ar/ACM-RemediarPublicaciones1.nsf/PubBoleWeb/1D38A9C8 4570D5430325726E0069CC25/\$File/Boletin 15.pdf. Acceso el 17 de octubre de 2008.

27. Bernztein R. El hierro, la anemia y el uso racional en el Programa Remediar. Atención Primaria de la Salud. Boletín PROAPS-REMEDIAR 2003;3(4):4-6. Hallado en http://www. remediar.gov.ar / ACM-RemediarPublicaciones1.nsf/PubBoleWeb/7D7A97AE 2D0939C50325726E0068E976/\$File/Boletin 4.pdf. Acceso el 17 de septiembre de 2008.

Manuscrito recibido el 17 de septiembre de 2007. Aceptado para publicación, tras revisión, el 30 de junio de 2008. 
ABSTRACT Objectives. To estimate the direct and indirect cost of iron deficiency (ID) and irondeficiency anemia (IDA) in Argentina and compare it with the cost of a prevention and treatment program.

Cost-benefit of a prevention and treatment program to reduce iron deficiency in Argentina

Methods. Analysis of a prior scenario to gage the relative cost-benefit of an IDA prevention and treatment program for all low-income children and expectant mothers without social coverage/benefits in Argentina. The economic consequences of ID and IDA were estimated as direct (cost of care for premature birth) and indirect costs (future lost productivity due to poor cognitive development due of children with ID and current reduced productivity of adults with IDA) employing the specific methodology designed by The Micronutrient Initiative (Ottawa, Canada). The interventions were defined according the practical clinical guidelines in use in Argentina and the item costs were taken from Ministry of Health price lists.

Results. Each US\$ 1.00 invested in an ID and IDA prevention and treatment program, assuming 90\% coverage of breastfeeding/pregnant uninsured low-income mothers, would save US\$33.40 by preventing the economic losses that would otherwise result from these conditions.

Conclusions. DH interventions not only significantly improve the health status of the population, but also offer a considerable savings.

Key words Anemia; anemia, iron-deficiency; iron deficiency; dietary supplements; Argentina. 